\title{
Statistical analysis of Rayleigh competing risks model based on partially step stress Type-II censoring samples
}

\author{
Abdullah M. Almarashi ${ }^{\mathrm{a}, *}$, Ali Algarni ${ }^{\mathrm{a}}$, G. A. Abd-Elmougod ${ }^{\mathrm{b}}$, Sayed Abdel-Khalek ${ }^{\mathrm{c}}$ \\ a Statistic Department, Faculty of Science, King Abdulaziz University, Jeddah, Saudi Arabia. \\ ${ }^{b}$ Mathematics department, Faculty of Science, Taif University, Taif, Saudi Arabia. \\ ${ }^{c}$ Mathematics department, Faculty of Science, Sohag University, Sohag, Egypt.
}

\begin{abstract}
This paper, discusses the problem of partially step-stress ALTs (accelerated life tests) form Rayleigh competing risks model. Type-II censored scheme is used in obtaining the observed censoring data. The method of MLE (maximum likelihood estimation) of the model parameters for point and approximate confidence intervals are considered. Also, bootstrap confidence intervals of model parameters are discussed. Simulation study is adopted to assess and compare our proposed method. Finally, some comment to illustrate the behavior of numerical results.
\end{abstract}

Keywords: Competing risk model, accelerate life test, Rayleigh distribution, maximum likelihood estimations, bootstrap confidence intervals.

2010 MSC: 60E05, 62E10, 62N05.

(C)2019 All rights reserved.

\section{Introduction}

The partially step-stress ALTs is a special case of ALTs, in which the experimenter allows to change the stress levels during the experiment. The stress levels will be changed at prior pre-fixed time. The data will be collected from such a partial step-stress ALTs, may then will be used to estimate the underlying model of failure times under use stress level. Several works are presented in this context, Balakrishnan [3], Balakrishnan et al. [5] and Balakrishnan and Xie [4]. The optimal step-stress test under discussed by Guan and Tang [10] and Lin and Chou [15]. Recently, inference of step-stress model presented by David and Kundu [13] and Soliman et all [19]. In a different area of reliable statistics, failure time of the units associated with one or more fatal risk factor. It is necessary to assess one risk factors with respect to other risk factor. This model is called competing risks model, in which observation is given in the bivariate form, time to failure and it is the cause of failure, some investigated the competing risks models in Crowder [6]. Different work exposed to lifetime distributions, with a competing risk model for example, Park [16], Kunduand Sarhan [14], Sarhan [17], Sarhan et al. [18], and Bakobanand Abd-Elmougod [2]. In reliability

\footnotetext{
*Corresponding Author

Email address: aalmarashi@kau.edu.sa (Abdullah M. Almarashi)

doi: $10.22436 /$ jnsa.012.04.04 
experiments, for consideration of time and cost the censoring is common phenomenon. Type-I censoring as well as Type-II censoring are a common censoring schemes in life test experiments. The experiment in Type-I censoring terminates at a pre-fixed time point, but the experiment in Type-II censoring at a pre-fixed number of failure.

The competing risks model with two different risk factors under Type-II censoring sample is described as follows. Suppose that, the life test experiment beginning with independent units, each of time and cause of failure $\left(T_{i ; m, n}, \delta_{i}\right)$ where $\delta_{i} \in\{1,2\}$ has recorded until $\left(T_{m ; m, n}, \delta_{i}\right)$, where $m \leqslant n$. The observed sample $\left(T_{i ; m, n}, \delta_{i}\right), i=1,2, \ldots, m$ are called Type-II competing risks sample.

The likelihood function of the observed sample $\left(T_{i ; m, n}, \delta_{i}\right), i=1,2, \ldots, m$, is given by

$$
\begin{aligned}
\mathrm{L}(\Theta \mid \mathrm{T}) & =\mathrm{C} \prod_{i=1}^{\mathrm{m}}\left[\mathrm{h}_{1}\left(\mathrm{t}_{i ; m, n}\right)\right]^{\eta\left(\delta_{i}=1\right)}\left[\mathrm{h}_{2}\left(\mathrm{t}_{i ; m, n}\right)\right]^{\eta\left(\delta_{i}=2\right)} \times\left[\mathrm{S}_{1}\left(\mathrm{t}_{\mathrm{m} ; \mathrm{m}, \mathrm{n}}\right) S_{2}\left(\mathrm{t}_{\mathrm{mm}, \mathrm{n}}\right)\right]^{(\mathrm{n}-\mathrm{m})}, \\
0 & <\mathrm{t}_{1 ; \mathrm{m}, \mathrm{n}}<\mathrm{t}_{2 ; \mathrm{m}, \mathrm{n}}<\cdots<\mathrm{t}_{\mathrm{m} ; \mathrm{m}, \mathrm{n}}<\infty,
\end{aligned}
$$

where $C=\frac{n !}{(n-m) !}, S()=.1-F(),. h=\frac{f(.)}{F(.)}, \Theta$ is the model parameters vector, and

$$
\eta\left(\delta_{i}=j\right)= \begin{cases}1, & \delta_{i}=j, \\ 2, & \delta_{i} \neq 2 .\end{cases}
$$

Our objective, is analyzing the competing risks model of partially step-stress ALTs under Type-II censoring sample from Rayleigh lifetime random variable. The estimation procedures with the ML method of the model parameters and accelerated factor is discussed. Also, the two, approximate information matrix and bootstrap techniques are used to construct different confidence intervals of the Rayleigh parameters and accelerated factor. The performances of the point estimates are measured in terms of mean estimate and MSE (mean squared error) and interval estimates measured in terms of mean length and probability coverage through Monte Carlo simulation .

This paper is described as, the model formulation of the partially step-stress ALTs under Type-II censoring and independently Rayleigh lifetime random variables of the risk factors in Section 2 . The MLEs of Rayleigh parameters and accelerated factor as well as asymptotic confidence intervals, are adopted in Section 3. Also, in Section 4, we presented a parametric bootstrap confidence intervals. In Section 5, the quality of points and intervals estimations are measured in terms of MSE and PC (probability coverage) via Monte Carlo study. Some comments are presented in Section 6.

\section{Model formulation and notation}

Under the assumption that, there are only two causes of failure are exist. We describe different notations we are going to use in this paper.

For $i^{\text {th }}$ unit, the lifetime random variable is presented by $T_{i}$.

For $i^{\text {th }}$ unit and the cause $j, j=1,2$, the lifetime random variable is presented by $T_{i j}$.

The cdf (cumulative distribution function) of $T_{i}$ is presented by: $F($.$) .$

The pdf (probability density function) of $F($.$) is presented by f($.$) .$

The cdf of $T_{i j}$ is presented by $F_{j}($.$) .$

The pdf of $F_{i j}($.$) is presented by f_{j}($.$) .$

The survival function of $T_{i j}$ is presented by $S_{j}($.$) .$

For $i^{\text {th }}$ unit failure the cause of failure has the indicator presented by $\delta_{i}$.

Let $n$ identical units are tested under the use condition and each of failure times and risk factor caused failure are recorded. At the prior fixed time $\tau$, the units are tested under stress condition, then the test runs until a prior fixed number of failures $m$ is observed. Under consideration that, the two cause of failure and the failure time has an independent Rayleigh distribution. To simplify the notation we will use henceforth $T_{i}$ instead of $T_{i: m: n}, i=1,2, \ldots, m$. The model studied in the paper satisfies the following assumptions. 
I) The lifetime of the unit is denoted as $T_{i}, i=1,2, \ldots, m$. The time at which the unit $i$ fails due to cause $j$ is $T_{j i}$, and $T_{i}=\min \left\{T_{1 i}, T_{2 i}\right\}$.

II) The distribution of the random variable $T_{j i}$ is is Rayleigh distribution with scale $\beta_{j}, j=1,2$ and $i=1,2, \cdots, m$. That is, the pdf and cdf, given by

$$
f_{j 1}(t)=2 \beta_{j} t e^{-\beta_{j} t^{2}}, \quad i=1,2 ., t>0, \beta_{j}>0, \quad F_{j 1}(t)=1-e^{-\beta_{j} t^{2}} .
$$

The corresponding reliability and failure rate functions of this distribution at some $t$, are given, respectively by

$$
S_{j 1}(t)=e^{-\beta_{j} t^{2}}, t>0, \quad h_{j 1}(t)=2 \beta_{j} t, t>0 .
$$

A wide applications of the Rayleigh distribution, is in communication engineering, and several applications in life tests experiment. Rayleigh distribution is considered as a special case of the Weibull distribution. Also, for more about life testing of electrovacum devices and recently, more information about Rayleigh lifetime distribution see Al-Matrafi and Abd-Elmougod [1].

III) The shorten from the lifetime of test units is appeared to multiply the remaining lifetime of the unit by the inverse of the accelerated factor. The total lifetime of a test unit, denoted by $Y$, defined under, use and accelerated conditions. So, the lifetime of the unit in partially step-stress ALTs, is define by

$$
Y= \begin{cases}T, & T<\tau, \\ \tau+\lambda^{-1}(T-\tau), & T>\tau,\end{cases}
$$

where the parameter $\lambda$ present the accelerated factor, $\tau$ is the time at which stress is changed to higher level and the lifetime of unit $\mathrm{T}$ computed at use condition. Under consideration that Rayleigh lifetime distribution of units with parameters $\beta_{j}$. Then, lifetime $Y$ of an unit has the pdf presented by.

$$
f_{j}(y)= \begin{cases}f_{j 2}(y), & y>\tau, \\ f_{j 1}(y), & 0<y \leqslant \tau, \\ 0, & y<0\end{cases}
$$

where

$$
f_{j 2}(y)=2 \lambda \beta_{j}(\tau+\lambda(y-\tau)) e^{-\beta_{j}(\tau+\lambda(y-\tau))^{2}},
$$

and $f_{j 1}(y)$ is given by $(2.1)$.

(2.3) is obtained from (2.1) after transformation variable from (1.1) and (2.2). The cdf, reliability function $S_{j 2}(y)$, and hazard rate function $h_{j 2}(y)$, are respectively presented by

$$
F_{j 2}(x)=1-e^{-\beta_{j}(\tau+\lambda(y-\tau))^{2}}, \quad S_{j 2}(t)=e^{-\beta_{j}(\tau+\lambda(y-\tau))^{2}}, \quad \text { and } \quad h_{j 2}(t)=2 \lambda \beta_{j}(\tau+\lambda(y-\tau)) .
$$

The total test is terminated respected to Type-II censoring when the number of failure is reached to $\mathrm{m}<\mathrm{n}$. The observed values of the total lifetime $Y$ are $\left(y_{1}, \delta_{1}\right)<\left(y_{2}, \delta_{2}\right)<\cdots<\left(y_{J}, \delta_{J}\right)<\tau<\left(y_{J+1}, \delta_{J+1}\right)<\cdots<$ $\left(y_{m}, \delta_{m}\right)$, where $J$ is the number of units failed at normal conditions and $m-J$ at accelerated conditions. The likelihood function of $\left(y_{1}, \delta_{1}\right)<\left(y_{2}, \delta_{2}\right)<\cdots<\left(y_{J}, \delta_{J}\right)<\tau<\left(y_{J+1}, \delta_{J+1}\right)<\cdots<\left(y_{m}, \delta_{m}\right)$ with consideration that $\tau<y_{m}$, is given by

$$
\begin{aligned}
L(\Theta \mid Y)= & C \prod_{i=1}^{J}\left[h_{11}\left(y_{i}\right)\right]^{I\left(\delta_{i}=1\right)}\left[h_{21}\left(y_{i}\right)\right]^{I\left(\delta_{i}=2\right)} \prod_{i=J+1}^{m}\left[h_{12}\left(t_{i}\right)\right]^{I\left(\delta_{i}=1\right)}\left[h_{22}\left(t_{i}\right)\right]^{I\left(\delta_{i}=2\right)} \\
& \times\left[S_{21}\left(y_{m}\right) S_{22}\left(y_{m}\right)\right]^{(n-m)}, \\
0< & \left(y_{1}, \delta_{1}\right)<\left(y_{2}, \delta_{2}\right)<\ldots<\left(y_{J}, \delta_{J}\right)<\tau<\left(y_{J+1}, \delta_{J+1}\right)<\ldots<\left(y_{m}, \delta_{m}\right)<\infty,
\end{aligned}
$$

where $\mathrm{C}$ is given in (1.1). 


\section{Maximum likelihood estimation}

Let the original sample $\left(y_{1}, \delta_{1}\right)<\left(y_{2}, \delta_{2}\right)<\cdots<\left(y_{J}, \delta_{J}\right)<\tau<\left(y_{J+1}, \delta_{J+1}\right)<\cdots<\left(y_{m}, \delta_{m}\right)$ be Rayleigh random variables with parameters $\beta_{j}$ for $j=1,2$. The joint likelihood function of (2.4), reduces to the following function

$$
L\left(\lambda, \beta_{1}, \beta_{2} \mid \underline{y}\right)=\beta_{1}^{m_{1}} \beta_{2}^{m_{2}} \lambda^{m-J} e^{-(n-m)\left(\beta_{1}+\beta_{1}\right)\left(\tau+\lambda\left(y_{m}-\tau\right)\right)^{2}} \prod_{i=1}^{J} y_{i} \prod_{i=J+1}^{m}\left(\tau+\lambda\left(y_{i}-\tau\right)\right),
$$

where $m_{1}$ and $m_{2}$ are the number of units failure with cause 1 and 2, respectively. The log-likelihood function is written as follows,

$$
\begin{aligned}
\ell\left(\lambda, \beta_{1}, \beta_{2} \mid \underline{y}\right)= & m_{1} \log \beta_{1}+m_{2} \log \beta_{2}+(m-J) \log \lambda+\sum_{i=1}^{J} \log y_{i}+\sum_{i=J+1}^{m} \log \left(\tau+\lambda\left(y_{i}-\tau\right)\right) \\
& -(n-m)\left(\beta_{1}+\beta_{1}\right)\left(\tau+\lambda\left(y_{m}-\tau\right)\right)^{2} .
\end{aligned}
$$

Equations (3.1) reduce to, after taken the first partial derivatives to $\beta_{1}$ and $\beta_{2}$, to the likelihood equations presented by

$$
\begin{aligned}
& \frac{\partial \ell\left(\lambda, \beta_{1}, \beta_{2} \mid \underline{y}\right)}{\partial \beta_{1}}=\frac{m_{1}}{\beta_{1}}-(n-m)\left(\tau+\lambda\left(y_{m}-\tau\right)\right)^{2}=0, \\
& \frac{\partial \ell\left(\lambda, \beta_{1}, \beta_{2} \mid \underline{y}\right)}{\partial \beta_{2}}=\frac{m_{2}}{\beta_{2}}-(n-m)\left(\tau+\lambda\left(y_{m}-\tau\right)\right)^{2}=0,
\end{aligned}
$$

hence from (3.2) and (3.3) we obtain the ML estimate of $\beta_{1}$ and $\beta_{2}$ as

$$
\begin{aligned}
& \hat{\beta}_{1}(\lambda)=\frac{m_{1}}{(n-m)\left(\tau+\lambda\left(y_{m}-\tau\right)\right)^{2}}, \\
& \hat{\beta}_{2}(\lambda)=\frac{m_{2}}{(n-m)\left(\tau+\lambda\left(y_{m}-\tau\right)\right)^{2}} .
\end{aligned}
$$

Also the derivatives of (3.1) to $\lambda$ reduce to

$$
\frac{\partial \ell\left(\lambda, \beta_{1}, \beta_{2} \mid \underline{y}\right)}{\partial \lambda}=\frac{(m-J)}{\lambda}+\sum_{i=J+1}^{m} \frac{\left(y_{i}-\tau\right)}{\tau+\lambda\left(y_{i}-\tau\right)}-2(n-m)\left(\beta_{1}+\beta_{1}\right)\left(y_{m}-\tau\right)\left(\tau+\lambda\left(y_{m}-\tau\right)\right)=0 .
$$

From (3.4) and the value of parameters in (3.2) and (3.3), we obtain the single nonlinear equation of $\lambda$, as follows

$$
\frac{(m-J)}{\lambda}+\sum_{i=J+1}^{m} \frac{\left(y_{i}-\tau\right)}{\tau+\lambda\left(y_{i}-\tau\right)}-2\left(m_{1}+m_{2}\right) \frac{\left(y_{m}-\tau\right)}{\left(\tau+\lambda\left(y_{m}-\tau\right)\right)}=0
$$

Using (3.2), (3.3), and (3.1), we obtain

$$
\begin{aligned}
g(\lambda)= & m_{1} \log \left[\frac{m_{1}}{(n-m)\left(\tau+\lambda\left(y_{m}-\tau\right)\right)^{2}}\right]+m_{2} \log \left[\frac{m_{2}}{(n-m)\left(\tau+\lambda\left(y_{m}-\tau\right)\right)^{2}}\right] \\
& +(m-J) \log \lambda+\sum_{i=1}^{J} \log y_{i}+\sum_{i=J+1}^{m} \log \left(\tau+\lambda\left(y_{i}-\tau\right)\right)-\left(m_{1}+m_{2}\right) .
\end{aligned}
$$

Therefore, the MLE $\hat{\lambda}$ of $\lambda$, from (3.5) with respect to $\lambda$. Using Newton Raphson method or the same arguments in Gupta and Kundu [11] with initial point obtained from the plot of profile log-likelihood function given in (3.6).

\subsection{Approximate confidence intervals}

From the log-likelihood function given in (3.1) after taking the second derivatives, we obtain the 
following equations

$$
\begin{aligned}
& \frac{\partial^{2} L\left(\lambda, \beta_{1}, \beta_{2} \mid \underline{y}\right)}{\partial \beta_{1}^{2}}=-\frac{m_{1}}{\beta_{1}^{2}} \\
& \frac{\partial^{2} L\left(\lambda, \beta_{1}, \beta_{2} \mid \underline{y}\right)}{\partial \beta_{2}^{2}}=-\frac{m_{2}}{\beta_{2}^{2}} \\
& \frac{\partial^{2} L\left(\lambda, \beta_{1}, \beta_{2} \mid \underline{y}\right)}{\partial \lambda^{2}}=\frac{-(m-J)}{\lambda^{2}}-\sum_{i=J+1}^{m} \frac{\left(y_{i}-\tau\right)^{2}}{\left(\tau+\lambda\left(y_{i}-\tau\right)\right)^{2}}-2(n-m)\left(\beta_{1}+\beta_{1}\right)\left(y_{m}-\tau\right)^{2}, \\
& \frac{\partial^{2} L\left(\lambda, \beta_{1}, \beta_{2} \mid \underline{y}\right)}{\partial \beta_{1} \partial \beta_{2}}=\frac{\partial^{2} L\left(\lambda, \beta_{1}, \beta_{2} \mid \underline{y}\right)}{\partial \beta_{2} \partial \beta_{1}}=0, \\
& \frac{\partial^{2} L\left(\lambda, \beta_{1}, \beta_{2} \mid \underline{y}\right)}{\partial \beta_{1} \partial \lambda}=\frac{\partial^{2} L\left(\lambda, \beta_{1}, \beta_{2} \mid \underline{y}\right)}{\partial \lambda \partial \beta_{1}}=-2(n-m)\left(y_{m}-\tau\right)\left(\tau+\lambda\left(y_{m}-\tau\right)\right)
\end{aligned}
$$

with some mild regularity conditions the MLEs of parameters $\beta_{1}, \beta_{1}$ and $\lambda$ is approximately Gaussian distribution with mean $\left(\beta_{1}, \beta_{1}, \lambda\right)$ and covariance matrix $\Sigma^{-1}\left(\beta_{1}, \beta_{1}, \lambda\right)$ where $\Sigma\left(\beta_{1}, \beta_{1}, \lambda\right)$ is the expectation of negative equations (3.7)-(3.11). Practice, $\Sigma^{-1}\left(\beta_{1}, \beta_{1}, \lambda\right)$ is estimated by $\Sigma_{0}^{-1}\left(\hat{\beta}_{1}, \hat{\beta}_{1}, \hat{\lambda}\right)$ where $\Sigma_{0}\left(\beta_{1}, \beta_{1}, \lambda\right)$ is the observed information matrix with inverse, given by

$$
\Sigma_{0}^{-1}\left(\hat{\beta}_{1}, \hat{\beta}_{1}, \hat{\lambda}\right)=\left[\begin{array}{c}
-\frac{\partial^{2} L\left(\lambda, \beta_{1}, \beta_{2} \mid \underline{y}\right)}{\partial \beta_{1}^{2}}-\frac{\partial^{2} L\left(\lambda, \beta_{1}, \beta_{2} \mid \underline{y}\right)}{\partial \beta_{1} \partial \beta_{2}}-\frac{\partial^{2} L\left(\lambda, \beta_{1}, \beta_{2} \mid \underline{y}\right)}{\partial \beta_{1} \partial \lambda} \\
-\frac{\partial^{2} L\left(\lambda, \beta_{1}, \beta_{2} \mid \underline{y}\right)}{\partial \beta_{2} \partial \beta 1}-\frac{\partial^{2} L\left(\lambda, \beta_{1}, \beta_{2} \mid \underline{y}\right)}{\partial \beta_{2}^{2}}-\frac{\partial^{2} L\left(\lambda, \beta_{1}, \beta_{2} \mid \underline{y}\right)}{\partial \beta_{2} \partial \lambda} \\
-\frac{\partial^{2} L\left(\lambda, \beta_{1}, \beta_{2} \mid \underline{y}\right)}{\partial \lambda \partial \beta 1}-\frac{\partial^{2} L\left(\lambda, \beta_{1}, \beta_{2} \mid \underline{y}\right)}{\partial \lambda \partial \beta_{2}}-\frac{\partial^{2} L\left(\lambda, \beta_{1}, \beta_{2} \mid y \underline{y}\right)}{\partial \lambda_{2}^{2}}
\end{array}\right]_{\left(\hat{\beta}_{1}, \hat{\beta}_{1}, \hat{\lambda}\right)}^{-1} .
$$

Hence, the Gaussian approximation is used as follows

$$
\left(\hat{\beta}_{1}, \hat{\beta}_{1}, \hat{\lambda}\right) \rightarrow N\left(\left(\beta_{1}, \beta_{1}, \lambda\right), \Sigma_{0}^{-1}\left(\hat{\beta}_{1}, \hat{\beta}_{1}, \hat{\lambda}\right)\right),
$$

The Gaussian distributed with mean $\left(\beta_{1}, \beta_{1}, \lambda\right)$ and variance covariance matrix $\Sigma_{0}^{-1}\left(\hat{\beta}_{1}, \hat{\beta}_{1}, \hat{\lambda}\right)$ is applied to obtain the approximate confidence intervals of $\beta_{1}, \beta_{1}$ and $\lambda$. Thus, the $100(1-2 \alpha) \%$ approximate confidence intervals of $\beta_{1}, \beta_{1}$, and $\lambda$ are given by

$$
\hat{\beta}_{1} \mp z_{\alpha} \sqrt{C_{11}}, \quad \hat{\beta}_{2} \mp z_{\alpha} \sqrt{C_{22}}, \text { and } \hat{\lambda}_{1} \mp z_{\alpha} \sqrt{C_{33}},
$$

respectively, where the elements $C_{11}, C_{22}$, and $C_{33}$ are taken from the diagonal of $\Sigma_{0}^{-1}\left(\hat{\beta}_{1}, \hat{\beta}_{1}, \hat{\lambda}\right)$ and $z_{\alpha}$ is the percentile right-tail with probable of $\alpha$ standard normal distribution.

\section{Bootstrap confidence intervals}

The estimations of confidence intervals, bias, ect., can be easily obtained with the bootstrap technique. Different types of bootstrap technique are available. The parametric bootstrap technique [7] and nonparametric bootstrap technique [9] are the common types of bootstrap technique. In the following, we expose to the parametric bootstrap algorithm that is adopted for obtaining interval bootstrap estimation, the percentile bootstrap [8], and bootstrap-t [12], confidence intervals .

1 From equations (3.2), (3.3), and (3.4) and the original Type-II sample, $\left(y_{1}, \delta_{1}\right)<\left(y_{2}, \delta_{2}\right)<\cdots<$ $\left(y_{J}, \delta_{J}\right)<\tau<\left(y_{J+1}, \delta_{J+1}\right)<\cdots<\left(y_{m}, \delta_{m}\right)$, the estimates $\hat{\beta}_{1}, \hat{\beta}_{2}$, and $\hat{\lambda}$ can be obtained.

2 Based on the estimates $\hat{\beta}_{1}, \hat{\beta}_{2}$, and $\hat{\lambda}$ and given prior values $m$ and $\tau$ in distributions (2.1) and (2.3), generate independent bootstrap samples $\left(\mathrm{y}_{1}^{*}, \delta_{1}^{*}\right)<\left(\mathrm{y}_{2}^{*}, \delta_{2}^{*}\right)<\cdots<\left(\mathrm{y}_{\mathrm{J}}^{*}, \delta_{\mathrm{J}}^{*}\right)<\tau<\left(\mathrm{y}_{\mathrm{J}+1}^{*}, \delta_{\mathrm{J}+1}^{*}\right)<\cdots<$ $\left(y_{m}^{*}, \delta_{m}^{*}\right)$.

3 Compute the bootstrap sample estimates $\hat{\beta}_{1}^{*}, \hat{\beta}_{2}^{*}$, and $\hat{\lambda}^{*}$ of $\hat{\beta}_{1}, \hat{\beta}_{2}$, and $\hat{\lambda}$ as in step 1 .

4 After repeating Steps 2 and $3 \mathrm{~S}$ times, then $S$ different bootstrap samples are represented.

5 For $\Psi^{*}=\left(\hat{\beta}_{1}^{*}, \hat{\beta}_{2}^{*}, \hat{\lambda}^{*}\right)$ put the bootstrap sample estimates in assiding order $\left(\Psi_{\mathrm{k}}^{*[1]}, \Psi_{\mathrm{k}}^{*[2]}, \ldots, \Psi_{\mathrm{k}}^{*[S]}\right), \mathrm{k}=$ $1,2,3$.

\subsection{Percentile bootstrap confidence intervals (PBCIs)}

The cdf of $\Psi_{\mathrm{k}}^{*}$ is given by $\mathrm{H}(\mathrm{y})=\mathrm{P}\left(\hat{\Psi}_{\mathrm{k}}^{*} \leqslant y\right)$. Then $\hat{\Psi}_{\mathrm{k}-\text { boot }}^{*}=\mathrm{H}^{-1}(\mathrm{y})$ for any given $\mathrm{y}$. The approximate 
bootstrap $100(1-2 \alpha) \%$ confidence interval of $\hat{\Psi}_{k}^{*}$ is given by

$$
\left[\hat{\Psi}_{\mathrm{k}-\text { bootp }}^{*}(\alpha), \hat{\Psi}_{\mathrm{k}-\text { bootp }}^{*}(1-\alpha)\right] \text {. }
$$

\subsection{Bootstrap-t confidence intervals (BTCI)}

From the relation

$$
\Phi_{k}^{*[j]}=\frac{\hat{\Psi}_{k}^{*[i]}-\hat{\Psi}_{k}}{\sqrt{\operatorname{var}\left(\hat{\Psi}_{k}^{*[i]}\right)}}, i=1,2, \ldots, S, k=1,2,3,
$$

where $\hat{\Psi}_{1}=\hat{\beta}_{1}, \hat{\Psi}_{2}=\hat{\beta}_{2}, \hat{\Psi}_{3}=\hat{\lambda}$. From the the order statistics $\Phi_{\mathrm{k}}^{*[1]}, \Phi_{\mathrm{k}}^{*[2]}, \ldots, \Phi_{\mathrm{k}}^{*[\mathrm{~S}]}$, we define the cumulative distribution $\mathrm{H}(\mathrm{y})=\mathrm{P}\left(\Phi_{\mathrm{k}}^{*}<y\right)$. For a given $y$, define

$$
\hat{\Psi}_{\mathrm{k}-\text { boot-t }}^{*}=\hat{\theta}_{\mathrm{l}}+\sqrt{\operatorname{Var}\left(\hat{\theta}_{\mathrm{l}}\right)} \mathrm{H}^{-1}(\mathrm{y}) \text {. }
$$

Then $100(1-2 \alpha) \%$ approximate confidence intervals of $\hat{\Psi}_{k}$ is given by

$$
\left(\hat{\Psi}_{k-\text { boot-t }}^{*}(\alpha), \hat{\Psi}_{k-\text { boot-t }}^{*}(1-\alpha)\right) \text {. }
$$

\section{Monte Carlo simulations}

The simulation study is built in this section, to compare and assess our developed theoretical results. Some numerical experiments performed for sample of sizes $n$, the effective sample sizes $m$, accelerated time $\tau$, and model parameters $\left(\beta_{1}, \beta_{1}, \lambda\right)$. We consider two cases separately.

(i) The model parameters $\left(\beta_{1}, \beta_{1}, \lambda\right)=(0.1,0.2,1.5)$ and accelerate time $\tau=(1.0,3.0,5.0)$.

(ii) The model parameters $\left(\beta_{1}, \beta_{1}, \lambda\right)=(0.5,0.7,2.0)$ and accelerate time $\tau=(0.5,1.0,1.5)$.

Simulation results are computed to compare the MLEs and bootstrap estimators, that mainly are compared in terms of their mean and MSE. The confidence intervals are compared in terms of their mean

\begin{tabular}{|c|c|c|c|c|c|c|c|c|c|c|c|c|c|}
\hline \multirow{3}{*}{$\tau$} & \multirow{3}{*}{$(n, m)$} & \multicolumn{6}{|c|}{ MLE } & \multicolumn{6}{|c|}{ Bootstrap } \\
\hline & & \multicolumn{3}{|c|}{ AVG } & \multicolumn{3}{|c|}{ MSE } & \multicolumn{3}{|c|}{ AVG } & \multicolumn{3}{|c|}{ MSE } \\
\hline & & $\beta_{1}$ & $\beta_{2}$ & $\lambda$ & $\beta_{1}$ & $\beta_{2}$ & $\lambda$ & $\beta_{1}$ & $\beta_{2}$ & $\lambda$ & $\beta_{1}$ & $\beta_{2}$ & $\lambda$ \\
\hline \multirow{5}{*}{1.0} & $(30,15)$ & 0.125 & 0.232 & 1.532 & 0.0885 & 0.0952 & 0.5542 & 0.136 & 0.241 & 1.555 & 0.0954 & 0.1233 & 0.6622 \\
\hline & $(30,25)$ & 0.121 & 0.239 & 1.530 & 0.0754 & 0.0840 & 0.4129 & 0.130 & 0.232 & 1.541 & 0.0821 & 0.1109 & 0.5219 \\
\hline & $(50,25)$ & 0.119 & 0.232 & 1.528 & 0.0749 & 0.0839 & 0.4091 & 0.131 & 0.230 & 1.543 & 0.0818 & 0.1080 & 0.5178 \\
\hline & $(50,40)$ & 0.121 & 0.222 & 1.526 & 0.0540 & 0.0814 & 0.3893 & 0.126 & 0.218 & 1.536 & 0.0742 & 0.0954 & 0.3295 \\
\hline & $(75,50)$ & 0.116 & 0.219 & 1.519 & 0.0519 & 0.0742 & 0.3860 & 0.122 & 0.216 & 1.524 & 0.0719 & 0.0900 & 0.3158 \\
\hline \multirow{5}{*}{3.0} & $(30,15)$ & 0.113 & 0.221 & 1.529 & 0.0864 & 0.0901 & 0.5472 & 0.132 & 0.233 & 1.545 & 0.0824 & 0.1198 & 0.6423 \\
\hline & $(30,25)$ & 0.114 & 0.218 & 1.514 & 0.0812 & 0.0821 & 0.5265 & 0.129 & 0.228 & 1.548 & 0.0800 & 0.1107 & 0.6318 \\
\hline & $(50,25)$ & 0.121 & 0.214 & 1.511 & 0.0801 & 0.0814 & 0.5249 & 0.130 & 0.219 & 1.543 & 0.0791 & 0.1088 & 0.5249 \\
\hline & $(50,40)$ & 0.120 & 0.213 & 1.510 & 0.0741 & 0.0795 & 0.4795 & 0.132 & 0.221 & 1.540 & 0.0762 & 0.1047 & 0.5209 \\
\hline & $(75,50)$ & 0.119 & 0.220 & 1.511 & 0.0720 & 0.0766 & 0.4788 & 0.129 & 0.222 & 1.539 & 0.0701 & 0.1002 & 0.5144 \\
\hline \multirow{5}{*}{5.0} & $(30,15)$ & 0.131 & 0.242 & 1.550 & 0.0987 & 0.1042 & 0.5987 & 0.154 & 0.261 & 1.560 & 0.0998 & 0.1252 & 0.6741 \\
\hline & $(30,25)$ & 0.128 & 0.235 & 1.542 & 0.0960 & 0.1011 & 0.5920 & 0.151 & 0.249 & 1.540 & 0.0961 & 0.1219 & 0.6041 \\
\hline & $(50,25)$ & 0.130 & 0.232 & 1.535 & 0.0940 & 0.1002 & 0.5908 & 0.144 & 0.248 & 1.523 & 0.0920 & 0.1200 & 0.5642 \\
\hline & $(50,40)$ & 0.128 & 0.226 & 1.534 & 0.0796 & 0.0821 & 0.5007 & 0.140 & 0.236 & 1.521 & 0.0865 & 0.0989 & 0.4523 \\
\hline & $(75,50)$ & 0.123 & 0.212 & 1.520 & 0.0701 & 0.0741 & 0.452 & 0.131 & 0.200 & 1.509 & 0.0652 & 0.0974 & 0.4185 \\
\hline
\end{tabular}
lengths (AL) and the probability coverage $(\mathrm{CP})$. The results in this paper are computed with Mathematica version 8 and reported in Tables 1-4.

Table 1: The mean estimates and MSEs for the parameters $\left(\beta_{1}, \beta_{2}, \lambda\right)$ at $(0.1,0.2,1.5)$. 
Table 2: The AL and (CP) of MLE, PBCIs and PTCIs of $95 \%$ CIs for the parameters $\left(\beta_{1}, \beta_{2}, \lambda\right)$ at $(0.1,0.2,1.5)$.

\begin{tabular}{|c|c|c|c|c|c|c|c|c|c|c|}
\hline \multirow{2}{*}{$\tau$} & \multirow{2}{*}{$(n, m)$} & \multicolumn{3}{|c|}{ MLE } & \multicolumn{3}{|c|}{ PBCIs } & \multicolumn{3}{|c|}{ PTCIs } \\
\hline & & $\beta_{1}$ & $\beta_{2}$ & $\lambda$ & $\beta_{1}$ & $\beta_{2}$ & $\lambda$ & $\beta_{1}$ & $\beta_{2}$ & $\lambda$ \\
\hline \multirow{10}{*}{1.0} & $(30,15)$ & 0.3954 & 0.5814 & 4.2145 & 0.4918 & 0.7124 & 5.2333 & 0.3690 & 0.5714 & 4.2092 \\
\hline & & $(0.88)$ & $(0.89)$ & $(0.90)$ & $(0.88)$ & $(0.88)$ & $(0.87)$ & $(0.90)$ & $(0.91)$ & (0.89) \\
\hline & $(30,25)$ & 0.3886 & 0.5790 & 4.2080 & 0.4900 & 0.7009 & 5.2301 & 0.3642 & 0.5650 & 4.2003 \\
\hline & & $(0.89)$ & $(0.90)$ & $(0.91)$ & $(0.89)$ & $(0.90)$ & $(0.89)$ & $(0.91)$ & $(0.91)$ & (0.92) \\
\hline & $(50,25)$ & 0.3870 & 0.5782 & 4.2083 & 0.4910 & 0.7004 & 5.2312 & 0.3641 & 0.5644 & 4.2001 \\
\hline & & $(0.88)$ & $(0.91)$ & $(0.93)$ & $(0.89)$ & $(0.91)$ & $(0.90)$ & $(0.92)$ & $(0.94)$ & (0.96) \\
\hline & $(50,40)$ & 0.3741 & 0.5700 & 4.2001 & 0.4880 & 0.6520 & 5.2210 & 0.3524 & 0.5498 & 4.0321 \\
\hline & & $(0.91)$ & $(0.91)$ & $(0.91)$ & $(0.90)$ & $(0.92)$ & (0.91) & (0.93) & $(0.95)$ & (0.93) \\
\hline & $(75,50)$ & 0.3521 & 0.5421 & 4.0092 & 0.4690 & 0.6321 & 5.1213 & 0.3109 & 0.5321 & 4.0009 \\
\hline & & (0.93) & $(0.92)$ & $(0.96)$ & $(0.91)$ & $(0.93)$ & $(0.93)$ & $(0.92)$ & $(0.94)$ & (0.94) \\
\hline \multirow{10}{*}{3.0} & $(30,15)$ & 0.3261 & 0.5351 & 3.9995 & 0.4521 & 0.6830 & 4.9800 & 0.3219 & 0.5314 & 3.9901 \\
\hline & & $(0.89)$ & $(0.90)$ & (0.91) & $(0.88)$ & (0.87) & (0.89) & (0.90) & $(0.91)$ & (0.90) \\
\hline & $(30,25)$ & 0.3199 & 0.5289 & 3.9874 & 0.4401 & 0.6723 & 4.9650 & 0.3188 & 0.5218 & 3.9841 \\
\hline & & $(0.89)$ & (0.91) & (0.91) & $(0.89)$ & (0.90) & (0.91) & (0.91) & $(0.93)$ & (0.92) \\
\hline & $(50,25)$ & 0.3180 & 0.5262 & 3.9849 & 0.4390 & 0.6700 & 4.9618 & 0.3152 & 0.5201 & 3.9822 \\
\hline & & $(0.90)$ & $(0.92)$ & (0.91) & $(0.90)$ & (0.90) & (0.92) & (0.93) & $(0.93)$ & (0.96) \\
\hline & $(50,40)$ & 0.3074 & 0.5123 & 3.9709 & 0.4265 & 0.6601 & 4.9555 & 0.3100 & 0.5001 & 3.9650 \\
\hline & & $(0.91)$ & (0.93) & $(0.92)$ & $(0.92)$ & $(0.91)$ & $(0.90)$ & $(0.94)$ & $(0.95)$ & (0.92) \\
\hline & $(75,50)$ & 0.3001 & 0.5088 & 3.9611 & 0.4202 & 0.6554 & 4.9501 & 0.2952 & 0.4129 & 3.9564 \\
\hline & & $(0.92)$ & $(0.91)$ & $(0.94)$ & $(0.91)$ & $(0.91)$ & $(0.91)$ & $(0.93)$ & $(0.94)$ & (0.94) \\
\hline \multirow{10}{*}{5.0} & $(30,15)$ & 0.4213 & 0.5992 & 4.2854 & 0.5214 & 0.8546 & 5.3695 & 0.38421 & 0.58219 & 4.2991 \\
\hline & & $(0.87)$ & $(0.88)$ & $(0.88)$ & $(0.88)$ & $(0.87)$ & (0.89) & $(0.89)$ & $(0.90)$ & (0.89) \\
\hline & $(30,25)$ & 0.4188 & 0.5940 & 4.2801 & 0.5178 & 0.8513 & 5.3665 & 0.3840 & 0.58201 & 4.2974 \\
\hline & & $(0.89)$ & $(0.90)$ & $(0.89)$ & $(0.89)$ & $(0.89)$ & $(0.90)$ & (0.91) & $(0.91)$ & (0.90) \\
\hline & $(50,25)$ & 0.4179 & 0.5932 & 4.2798 & 0.5162 & 0.8510 & 5.3666 & 0.3835 & 0.5820 & 4.2969 \\
\hline & & $(0.90)$ & $(0.90)$ & $(0.90)$ & $(0.91)$ & $(0.89)$ & (0.90) & $(0.92)$ & $(0.91)$ & (0.92) \\
\hline & $(50,40)$ & 0.4112 & 0.5889 & 4.2752 & 0.5114 & 0.8490 & 5.3608 & 0.3800 & 0.5791 & 4.2911 \\
\hline & & $(0.91)$ & $(0.92)$ & $(0.91)$ & $(0.92)$ & $(0.90)$ & (0.91) & (0.92) & $(0.93)$ & (0.96) \\
\hline & $(75,50)$ & 0.4019 & 0.5812 & 4.2701 & 0.5100 & 0.8460 & 5.3542 & 0.3762 & 0.5745 & 4.2889 \\
\hline & & $(0.92)$ & $(0.92)$ & $(0.93)$ & $(0.91)$ & $(0.91)$ & $(0.92)$ & $(0.91)$ & $(0.93)$ & (0.95) \\
\hline
\end{tabular}

Table 3: The mean estimates and MSEs for the parameters $\left(\beta_{1}, \beta_{2}, \lambda\right)$ at $(0.5,0.7,2.0)$.

\begin{tabular}{|c|c|c|c|c|c|c|c|c|c|c|c|c|c|}
\hline \multirow{3}{*}{$\tau$} & \multirow{3}{*}{$(n, m)$} & \multicolumn{6}{|c|}{ MLE } & \multicolumn{6}{|c|}{ Bootstrap } \\
\hline & & \multicolumn{3}{|c|}{ AVG } & \multicolumn{3}{|c|}{ MSE } & \multicolumn{3}{|c|}{ AVG } & \multicolumn{3}{|c|}{ MSE } \\
\hline & & $\beta_{1}$ & $\beta_{2}$ & $\lambda$ & $\beta_{1}$ & $\beta_{2}$ & $\lambda$ & $\beta_{1}$ & $\beta_{2}$ & $\lambda$ & $\beta_{1}$ & $\beta_{2}$ & $\lambda$ \\
\hline \multirow{5}{*}{0.5} & $(30,15)$ & 0.532 & 0.746 & 2.326 & 0.1423 & 0.2149 & 0.8642 & 0.556 & 0.779 & 2.353 & 0.2145 & 0.2362 & 0.8891 \\
\hline & $(30,25)$ & 0.528 & 0.742 & 2.219 & 0.1324 & 0.2084 & 0.8542 & 0.545 & 0.741 & 2.311 & 0.2102 & 0.2318 & 0.8831 \\
\hline & $(50,25)$ & 0.525 & 0.639 & 2.211 & 0.1315 & 0.2070 & 0.8533 & 0.540 & 0.733 & 2.302 & 0.2092 & 0.2301 & 0.8802 \\
\hline & $(50,40)$ & 0.521 & 0.636 & 2.203 & 0.1153 & 0.2001 & 0.7362 & 0.533 & 0.728 & 2.284 & 0.2042 & 0.2222 & 0.8741 \\
\hline & $(75,50)$ & 0.519 & 0.627 & 2.198 & 0.0987 & 0.1423 & 0.7105 & 0.522 & 0.725 & 2.214 & 0.1441 & 0.2210 & 0.8524 \\
\hline \multirow{5}{*}{1.0} & $(30,15)$ & 0.522 & 0.732 & 2.221 & 0.1012 & 0.2016 & 0.7521 & 0.5421 & 0.766 & 2.229 & 0.1458 & 0.2018 & 0.8258 \\
\hline & $(30,25)$ & 0.521 & 0.723 & 2.202 & 0.1000 & 0.2009 & 0.7501 & 0.5407 & 0.742 & 2.211 & 0.1400 & 0.1992 & 0.8211 \\
\hline & $(50,25)$ & 0.519 & 0.719 & 2.213 & 0.0984 & 0.2004 & 0.7498 & 0.5399 & 0.733 & 2.201 & 0.1328 & 0.1849 & 0.8011 \\
\hline & $(50,40)$ & 0.512 & 0.718 & 2.210 & 0.0920 & 0.1842 & 0.6231 & 0.5302 & 0.731 & 2.198 & 0.1142 & 0.1600 & 0.7012 \\
\hline & $(75,50)$ & 0.510 & 0.714 & 2.207 & 0.0911 & 0.1741 & 0.6124 & 0.5211 & 0.711 & 2.177 & 0.1147 & 0.1548 & 0.6321 \\
\hline \multirow{5}{*}{1.5} & $(30,15)$ & 0.555 & 0.765 & 2.342 & 0.1460 & 0.2189 & 0.8690 & 0.558 & 0.799 & 2.392 & 0.2177 & 0.2388 & 0.8911 \\
\hline & $(30,25)$ & 0.535 & 0.762 & 2.238 & 0.1340 & 0.2095 & 0.8559 & 0.547 & 0.743 & 2.322 & 0.2121 & 0.2332 & 0.8852 \\
\hline & $(50,25)$ & 0.534 & 0.761 & 2.236 & 0.1338 & 0.2092 & 0.8553 & 0.539 & 0.741 & 2.320 & 0.2118 & 0.2323 & 0.8847 \\
\hline & $(50,40)$ & 0.532 & 0.758 & 2.229 & 0.1300 & 0.2052 & 0.8539 & 0.536 & 0.730 & 2.309 & 0.2111 & 0.2300 & 0.8813 \\
\hline & $(75,50)$ & 0.524 & 0.744 & 2.224 & 0.1142 & 0.1754 & 0.8501 & 0.529 & 0.719 & 2.208 & 0.2006 & 0.2189 & 0.8610 \\
\hline
\end{tabular}


Table 4: The AL and (CP) of MLE, PBCIs and PTCIs of $95 \%$ CIs for the parameters $\left(\beta_{1}, \beta_{2}, \lambda\right)$ at $(0.1,0.2,1.5)$.

\begin{tabular}{|c|c|c|c|c|c|c|c|c|c|c|}
\hline \multirow{2}{*}{$\tau$} & \multirow{2}{*}{$(n, m)$} & \multicolumn{3}{|c|}{ MLE } & \multicolumn{3}{|c|}{ PBCIs } & \multicolumn{3}{|c|}{ PTCIs } \\
\hline & & $\beta_{1}$ & $\beta_{2}$ & $\lambda$ & $\beta_{1}$ & $\beta_{2}$ & $\lambda$ & $\beta_{1}$ & $\beta_{2}$ & $\lambda$ \\
\hline \multirow{10}{*}{0.5} & $(30,15)$ & 1.3951 & 1.9814 & 6.2143 & 1.5210 & 2.9012 & 6.998 & 1.3854 & 1.9621 & 6.2009 \\
\hline & & $(0.89)$ & $(0.90)$ & $(0.90)$ & $(0.88)$ & $(0.87)$ & $(0.89)$ & $(0.90)$ & $(0.91)$ & $(0.90)$ \\
\hline & $(30,25)$ & 1.3748 & 1.9621 & 5.2001 & 1.5128 & 2.8742 & 5.3965 & 1.3711 & 1.9598 & 5.0952 \\
\hline & & (0.90) & $(0.91)$ & $(0.91)$ & $(0.89)$ & $(0.90)$ & $(0.90)$ & $(0.92)$ & $(0.91)$ & (0.93) \\
\hline & $(50,25)$ & 1.3722 & 1.9627 & 5.1854 & 1.5109 & 2.8733 & 5.3949 & 1.3712 & 1.9580 & 5.0934 \\
\hline & & $(0.91)$ & $(0.89)$ & $(0.92)$ & $(0.90)$ & $(0.90)$ & $(0.91)$ & $(0.92)$ & $(0.93)$ & $(0.96)$ \\
\hline & $(50,40)$ & 1.3511 & 1.9490 & 5.1611 & 1.5002 & 2.8554 & 5.3711 & 1.3518 & 1.9487 & 5.0799 \\
\hline & & $(0.92)$ & $(0.91)$ & $(0.92)$ & $(0.92)$ & $(0.90)$ & $(0.91)$ & $(0.94)$ & $(0.94)$ & $(0.95)$ \\
\hline & $(75,50)$ & 1.3312 & 1.9385 & 5.1501 & 1.4854 & 2.8495 & 5.3621 & 1.3471 & 1.9400 & 5.0601 \\
\hline & & $(0.92)$ & $(0.92)$ & $(0.92)$ & $(0.92)$ & $(0.91)$ & $(0.92)$ & $(0.92)$ & $(0.97)$ & $(0.93)$ \\
\hline \multirow{10}{*}{1.0} & $(30,15)$ & 1.3900 & 1.9712 & 6.2095 & 1.5152 & 2.8541 & 6.9780 & 1.3756 & 1.9521 & 6.1247 \\
\hline & & $(0.88)$ & $(0.91)$ & $(0.89)$ & $(0.89)$ & $(0.89)$ & $(0.90)$ & $(0.91)$ & $(0.91)$ & (0.91) \\
\hline & $(30,25)$ & 1.3701 & 1.9590 & 5.1852 & 1.5065 & 2.8700 & 5.3901 & 1.3623 & 1.9502 & 5.0821 \\
\hline & & (0.90) & $(0.91)$ & $(0.91)$ & $(0.89)$ & $(0.90)$ & $(0.90)$ & $(0.92)$ & $(0.91)$ & (0.93) \\
\hline & $(50,25)$ & 1.3722 & 1.9582 & 5.1849 & 1.5058 & 2.8701 & 5.3889 & 1.3619 & 1.9501 & 5.0819 \\
\hline & & (0.91) & $(0.90)$ & $(0.92)$ & $(0.91)$ & $(0.90)$ & (0.91) & $(0.93)$ & $(0.93)$ & $(0.94)$ \\
\hline & $(50,40)$ & 1.3690 & 1.9501 & 5.1780 & 1.4985 & 2.8650 & 5.3801 & 1.3523 & 1.9458 & 5.0713 \\
\hline & & $(0.92)$ & $(0.91)$ & $(0.92)$ & $(0.92)$ & $(0.90)$ & $(0.93)$ & $(0.93)$ & $(0.94)$ & $(0.97)$ \\
\hline & $(75,50)$ & 1.3574 & 1.9482 & 5.1645 & 1.4750 & 2.8590 & 5.3774 & 1.3451 & 1.9362 & 4.9921 \\
\hline & & (0.93) & $(0.92)$ & $(0.92)$ & $(0.91)$ & (0.91) & (0.94) & $(0.96)$ & $(0.94)$ & (0.93) \\
\hline \multirow{10}{*}{1.5} & $(30,15)$ & 1.3988 & 1.9862 & 6.2170 & 1.5243 & 2.9060 & 7.1129 & 1.3882 & 1.9654 & 6.2032 \\
\hline & & $(0.88)$ & $(0.89)$ & $(0.90)$ & $(0.88)$ & $(0.87)$ & $(0.88)$ & $(0.89)$ & $(0.90)$ & $(0.90)$ \\
\hline & $(30,25)$ & 1.3775 & 1.9654 & 5.2039 & 1.5143 & 2.8767 & 5.3980 & 1.3733 & 1.9621 & 5.0984 \\
\hline & & $(0.89)$ & $(0.90)$ & $(0.91)$ & $(0.89)$ & $(0.91)$ & $(0.91)$ & $(0.91)$ & $(0.91)$ & $(0.92)$ \\
\hline & $(50,25)$ & 1.3772 & 1.9644 & 5.2034 & 1.5140 & 2.8759 & 5.3969 & 1.3729 & 1.9620 & 5.0979 \\
\hline & & $(0.92)$ & $(0.90)$ & $(0.92)$ & $(0.90)$ & $(0.90)$ & $(0.90)$ & $(0.93)$ & $(0.93)$ & $(0.94)$ \\
\hline & $(50,40)$ & 1.3522 & 1.9584 & 5.1629 & 1.5040 & 2.8570 & 5.3746 & 1.3553 & 1.9521 & 5.0814 \\
\hline & & $(0.90)$ & $(0.91)$ & $(0.93)$ & $(0.92)$ & $(0.91)$ & $(0.91)$ & $(0.92)$ & $(0.96)$ & $(0.95)$ \\
\hline & $(75,50)$ & 1.3322 & 1.9399 & 5.1536 & 1.4878 & 2.8511 & 5.3650 & 1.3497 & 1.9425 & 5.0633 \\
\hline & & $(0.91)$ & $(0.92)$ & $(0.92)$ & $(0.93)$ & $(0.91)$ & $(0.91)$ & $(0.93)$ & $(0.92)$ & $(0.92)$ \\
\hline
\end{tabular}

\section{Conclusions}

In this paper, we have discussed the Type-II censoring competing risks model in the presence of partially step stress ALT. Specially, we have proposed that the latent failure times under the competing risks follow independent Rayleigh distributions. The MLEs of the unknown model parameters are derived. We proposed the asymptotic distribution of MLEs and bootstrap method for constructing CIs. From the results in Tables 1-4 of the simulation study, some points are observed as follows.

(1): From the Tables 2 and 4, we observe that PTCIs performs the best as its CIs has a small length and coverage probabilities are much closer to the nominal levels than ACIs and PBCIs.

(2): From the Tables 1 and 2, we observe the point estimate of MLE performs the best than bootstrap estimates.

(3): From all tables, we observe that results for the value of accelerate change time $\tau$ performs the best for the value of $\tau$ close to distribution mean.

(4): For the effective $m$ effect sample size increases, the MSEs and the average length of different estimators are reduced.

\section{Acknowledgment}

This project was funded by the Deanship of Scientific Research (DSR) at King Abdulaziz University, 
Jeddah, under grant no. (G-438-130-38). The authors, therefore, acknowledge with thanks DSR for technical and financial support.

\section{References}

[1] B. N. Al-Matrafi, G. A. Abd-Elmougod, Statistical Inferences with Jointly Type-II Censored Samples From Two Rayleigh Distributions, Global J. Pure Appl. Math., 13 (2017), 8361-8372. 2

[2] R. A. Bakoban, G. A. Abd-Elmougod, MCMC in analysis of progressively first failure censored competing risks data for Gompertz model, J. Comput. Theor. Nanoscience, 13 (2016), 1-9. 1

[3] N. Balakrishnan, A synthesis of exact inferential results for exponential step-stress models and associated optimal accelerated life-tests, Metrika, 69 (2009), 351-396. 1

[4] N. Balakrishnan, Q. Xie, Exact inference for a simple step-stress model with Type-II hybrid censored data from the exponential distribution, J. Statist. Plann. Inference, 137 (2007), 2543-2563. 1

[5] N. Balakrishnan, Q. Xie, D. Kundu, Exact inference for a simple step-stress model from the exponential distribution under time constraint, Ann. Inst. Statist. Math., 61 (2009), 251-274. 1

[6] M. J. Crowder, Classical Competing Risks, Chapman \& Hall, Boca Raton, (2001). 1

[7] A. C. Davison, D. V. Hinkley, Bootstrap Methods and their Applications, Cambridge University Press, Cambridge, (1997). 4

[8] B. Efron, Censored data and bootstrap, J. Amer. Statist. Assoc., 76 (1981), 312-319. 4

[9] B. Efron, R. J. Tibshirani, An introduction to the bootstrap, Chapman and Hall, New York, (1993). 4

[10] Q. Guan, Y. Tang, Optimal step-stress test under Type-I censoring for multivariate exponential distribution, J. Statist. Plann. Inference, 142 (2012), 1908-1923. 1

[11] R. D. Gupta, D. Kundu, Generalized exponential distributions: statistical inferences, J. Stat. Theory Appl., 1 (2002), 101-118. 3

[12] P. Hall, Theoretical comparison of bootstrap confidence intervals, Ann. Statist., 16 (1988), 927-953. 4

[13] D. Han, D. Kundu, Inference for a step-stress model with competing risks for failure from the generalized exponential distribution under Type-I censoring, IEEE Tran. Reliab., 64 (2015), 31-43. 1

[14] D. Kundu, A. M. Sarhan, Analysis of incomplete data in the presence of competing risks among several groups, IEEE Tran. Reliab., 55 (2006), 262-271. 1

[15] C. T. Lin, C. C. Chou, Statistical inference for a lognormal step-stress model with Type-I censoring, IEEE Tran. Reliab., 61 (2012), 361-377. 1

[16] C. Park, Parameter estimation of incomplete data in competing risks using the EM algorithm, IEEE Tran. Reliab., 54 (2005), 282-290. 1

[17] A. M. Sarhan, Analysis of incomplete, censored data in competing risks models with generalized exponential distribution, IEEE Tran. Reliab., 56 (2007), 132-138. 1

[18] A. M. Sarhan, Analysis of incomplete, censored data in competing risks models with generalized Exponential distribution, IEEE Transactions on Reliability, 56 (2007), 132-138. 1

[19] A. A. Soliman, G. A. Abd-Elmougod, M. M. Al-Sobhi, Estimation in step-stress partially accelerated life tests for the Chen distribution using progressive Type-II censoring, Appl. Math. Infor. Sci., 11 (2017), 325-332. 1 